\title{
Vulnerabilidad de las especies de Dytiscidae (Coleoptera) en Cuba
}

\author{
Yoandri S. Megna ${ }^{1,2^{*}}$, David Sánchez-Fernández ${ }^{3}$, Ileana Fernández García ${ }^{4}$, \\ Bernardo Reyes-Tur ${ }^{2} \&$ Michael Balke ${ }^{5}$ \\ 1. Zoologische Staatssammlung, Münchhausenstraße 21, D-81247 München, Deutschland; ysmegna@gmail.com \\ 2. Departamento de Biología, Universidad de Oriente. Patricio Lumumba s/n, Santiago de Cuba 90500, Cuba; \\ breyestur@gmail.com \\ 3. Instituto de Ciencias Ambientales (ICAM). Universidad de Castilla-La Mancha. Campus Tecnológico de la Fábrica \\ de Armas, Toledo 45071, España; davidsan@um.es \\ 4. Instituto de Ecología y Sistemática, Carretera de Varona No. 11835 entre Oriente y Lindero, Reparto Parajón, \\ Municipio Boyeros, La Habana 19 C.P. 11900, Cuba; ileanafg@ecologia.cu \\ 5. SNSB-Zoologische Staatssammlung München, Münchhausenstrasse 21, 81247 München, Germany; \\ michael_balke@yahoo.de \\ * Correspondencia
}

Recibido 04-IV-2017. Corregido 08-I-2018. Aceptado 02-II-2018.

\begin{abstract}
Vulnerability of Dytiscidae species (Coleoptera) in Cuba. Cuba has one of the richest diving beetles species diversity in the Caribbean islands. However, Cuban Dytiscidae remain scarcely studied, and there is need to identify those species and habitats that urgently require effective conservation actions. Here we aim to identify the threatened taxa of the family Dytiscidae in Cuba according to their degree of vulnerability. For that, we compiled distributional data on the Cuban fauna, including data from literature, collections and own samplings carried out between the period 2000 and 2014. In addition, the vulnerability of the species was evaluated using a methodology that combines six criteria regarding both species and habitat attributes. This analysis of vulnerability was completed to $50(94 \%)$ out of 53 Cuban species. Five species $(9 \%)$ were identified as highly vulnerable, 20 (38\%) moderately and $25(48 \%)$ as having low conservation status. The five most threatened species were Laccodytes cobrinae, Laccophilus alariei, Copelatus barbouri, C. darlingtoni, and Desmopachria glabella. These species are endemic to Cuba and characterized by highly localized populations and low abundance as well as high habitat specificity. Thus, we propose the inclusion of these five species in both the red list of Cuban fauna and the IUCN Red List (under the category of "Vulnerable"). In addition, not all areas where these species were found belong to the National System of Protected Areas of Cuba. Thus, we suggest the inclusion of the Laguna Base Julio A. Mella and the North of Imías, and to develop effective management measures to prevent the alteration of these aquatic ecosystems. Rev. Biol. Trop. 66(2): 709-721. Epub 2018 June 01.
\end{abstract}

Key words: Adephaga, diving beetles, threatened species, IUCN, biodiversity.

En el actual contexto de cambio global, los ecosistemas experimentan un incremento de la intensidad del impacto humano a nivel mundial, como consecuencia tiene lugar una pérdida de biodiversidad sin precedentes (Ceballos et al., 2015). Esta pérdida de biodiversidad es más acusada en ambientes acuáticos interiores (Sánchez-Fernández et al., 2008), ya que a pesar de ser hábitats que contienen una cantidad de especies desproporcionadamente alta en relación a la superficie que ocupan (casi el $6 \%$ de las especies descritas, cubren sólo el $0.8 \%$ de la superficie mundial), se encuentran muy alterados por la actividad humana, lo que ha hecho que se reconozcan como uno de los ambientes más amenazados del mundo (Strayer, 2006).

En este contexto de crisis biológica, y ante un escenario de escasez de recursos para la conservación de la biodiversidad, se hace 
necesario identificar aquellas especies y hábitats que requieren con mayor urgencia medidas de conservación eficaces. Tradicionalmente, las prioridades de conservación a nivel de especie se basan en las denominadas "listas rojas", mediante la clasificación de los taxones en función de su grado de amenaza o probabilidad de extinción. Este tipo de documentos, por norma general, facilitan la elaboración de la legislación internacional, nacional y regional en materia de protección de especies.

A escala global, la Unión Internacional para la Conservación de la Naturaleza (UICN) proporciona un riguroso rango de categorías para clasificar las especies de acuerdo a su probabilidad de extinción en un determinado período de tiempo (IUCN, 2017). Estas categorías han sido ampliamente aceptadas en todo el mundo y forman la base de la Lista Roja de la UICN. Sin embargo, en base a los criterios utilizados en la misma, muchas especies de insectos quedarían enmarcadas en la categoría "Datos Insuficientes", siendo más que previsible que muchos de estos taxones tampoco contarán, a mediano plazo, con datos suficientes para abordar una clasificación rigurosa (Cardoso, Borges, Triantis, Ferrández, \& Martín, 2011). Quizás como consecuencia de lo anterior, sólo el $8 \%$ de las especies animales evaluadas como "amenazadas" (esto es, en peligro crítico, en peligro o vulnerables) en la Lista Roja de la UICN son insectos, frente al $66 \%$ de vertebrados, a pesar de que se conocen más de un millón de especies de insectos y sólo unas 65000 de vertebrados (IUCN, 2012).

Los coleópteros destacan por presentar una apreciable diversidad de formas, tamaños y estrategias ecológicas, no superadas por otro orden de insectos. En el caso de los coleópteros acuáticos, se consideran agentes eficaces para el control natural de larvas de mosquitos (Lundkvist, Ladin, Jackson, \& Svensson, 2003). Además, son importantes bioindicadores de los cambios espaciales y temporales producidos en los ecosistemas acuáticos (Benetti \& Garrido, 2010). En concreto, en Cuba se encuentra la mayor diversidad de especies de la familia Dytiscidae del conjunto de las Antillas, con
53 especies de las cuales aproximadamente el $23 \%$ son endémicas (Megna \& Sánchez-Fernández, 2016). Recientemente, se ha empezado a estudiar aspectos de taxonomía y faunística de estas especies (Megna, Deler-Hernández, \& Challet, 2011; Megna \& Epler, 2012; Megna \& Sánchez-Fernández, 2014). Sin embargo, se hace necesario que estos estudios puedan ser utilizados para realizar una adecuada legislación, gestión y ordenamiento del territorio, que permita la conservación de la biodiversidad. Por lo tanto, el presente estudio tiene como objetivo la clasificación de las especies de la familia Dytiscidae en Cuba en función de su grado de vulnerabilidad.

\section{MATERIALES Y MÉTODOS}

Área de estudio: El archipiélago cubano está formado por más de 1600 islas, islotes y cayos, ubicados entre los $23^{\circ} 17^{\prime} 09^{\prime \prime}-19^{\circ} 49^{\prime} 36^{\prime \prime}$ $\mathrm{N}-74^{\circ} 07^{\prime} 55^{\prime \prime}-84^{\circ} 57^{\prime} 54^{\prime \prime} \mathrm{W}$, con una extensión total de $109886 \mathrm{~km}^{2}$, rodeado por cuatro grupos insulares: Los Colorados y Sabana-Camagüey (al norte) y Jardines de la Reina y Los Canarreos (al sur). Este último archipiélago posee, la isla más extensa después de la Isla de Cuba, nombrada Isla de la Juventud, con 2419 $\mathrm{km}^{2}$ (Comisión Nacional de Nombres Geográficos, 2000). El archipiélago cubano limita al norte con el Estrecho de la Florida, el Canal de San Nicolás y el Canal Viejo de Bahamas; al este con el Paso de los Vientos; al sur con el Mar Caribe y el Estrecho de Colón; y al oeste, con el Estrecho de Yucatán. La situación geográfica del país, así como su pasado geológico, determinan las particularidades de su flora y su fauna (CNAP, 2009).

Obtención de la información faunística: La composición taxonómica y la distribución de los ditíscidos en Cuba fue determinada a partir de los datos recogidos en Megna y SánchezFernández (2016), que incluyen muestreos propios entre los años 2000 y 2014, citas de recopilación bibliográfica (desde 1856) y revisión de seis colecciones entomológicas tanto públicas como privadas (acrónimo: nombre de 
la colección): BSC-E: Colección Zoológica del Departamento de Zoología (BSC-E) del Centro Oriental de Ecosistemas y Biodiversidad (BIOECO), Santiago de Cuba, Cuba; CZCTR: Museo de Historia Natural "Charles Ramsden", Santiago de Cuba, Cuba; IES: Colección del Instituto de Ecología y Sistemática, La Habana, Cuba; CMT: Colección Mario Toledo, Parma, Italia; FSCA: Colección de Artrópodos del Estado de la Florida, Gainesville, Florida, EEUU; USNM: Museo Nacional de Historia Natural de los Estados Unidos, Washington, DC, EEUU.

\section{Tipos de hábitats ocupados por las espe-} cies de Dytiscidae en Cuba: Las estaciones en las que aparecieron los ditíscidos fueron clasificadas en diferentes tipos de hábitats según sus características fisionómicas, en cuanto al carácter dinámico o estático del agua (ambientes lóticos o lénticos), su persistencia en el tiempo (ambientes permanentes o temporales), la anchura y profundidad del cauce y la altitud. En total fueron identificados 11 tipos de hábitats (Cuadro 1). Posteriormente, se calculó el número de estaciones de muestreo asignadas a cada tipo de hábitat, y el número de cuadrículas de $2.5 \mathrm{~min}$ (aproximadamente $4.4 \times 4.4 \mathrm{~km}$ $=19.4 \mathrm{~km}^{2}$ en el Ecuador) en los que aparece cada tipo de hábitat. En total en Cuba existen 6 297 cuadrículas (Fig. 1).

Evaluación de la vulnerabilidad: La vulnerabilidad de las especies fue evaluada por

CUADRO 1

Tipos de hábitats utilizados para la evaluación de la vulnerabilidad de las especies de la familia Dytiscidae en Cuba. Se indica el número de localidades de muestreo (Loc) y de cuadrículas (Cuad) en las que aparece cada tipo de hábitat $(2.5 \times 2.5$ minutos $)$

TABLE 1

Types of habitats used to assess the vulnerability of species of the family Dytiscidae in Cuba. The number of sampling localities (Loc) and grids (Cuad) in which each type of habitat ( $2.5 \times 2.5$ minutes) is shown

\begin{tabular}{|c|c|c|c|c|}
\hline $\mathrm{N}^{\mathrm{o}}$ & Tipos de hábitats & Descripción & Loc & Cuad \\
\hline 1 & Arroyos de cabecera & $\begin{array}{l}\text { Constituye la parte superior o naciente de los ríos, con cauce estrecho } \\
(1.5 \mathrm{~m}) \text {, poco profundo }(0.5 \mathrm{~m}) \text {, de agua muy fría, transparente y sin } \\
\text { material de suspensión evidente. }\end{array}$ & 42 & 28 \\
\hline 2 & Tramos medios de los ríos & $\begin{array}{l}\text { Hábitats lóticos de agua dulce, de hasta } 1 \mathrm{~m} \text { de profundidad media y } \\
\text { con un ancho de entre } 1.5 \text { y } 5 \mathrm{~m} \text {. Puede estar influido por la presencia } \\
\text { de zonas urbanas relativamente próximas. }\end{array}$ & 31 & 20 \\
\hline 3 & Tramos bajos de los ríos & $\begin{array}{l}\text { Zona baja del tramo de un río, cercana a la desembocadura y donde la } \\
\text { pendiente es paulatinamente menor, con profundidad media superior a } \\
1 \mathrm{~m} \text { y ancho superior a los } 5 \mathrm{~m} \text {. Con frecuencia se encuentran influidos } \\
\text { por la actividad desarrollada en zonas urbanas próximas. }\end{array}$ & 11 & 8 \\
\hline 4 & Estuarios de los ríos & Hábitats lóticos con agua salobre en la desembocadura. & 1 & 1 \\
\hline 5 & Lagunas estuarinas & $\begin{array}{l}\text { Hábitats lénticos de agua salobre cercanos a la costa, que tienen libre } \\
\text { comunicación con el mar. }\end{array}$ & 1 & 1 \\
\hline 6 & Lagunas & Cuerpos de agua lénticos naturales, permanentes. & 57 & 16 \\
\hline 7 & Charcas & Cuerpos de agua lénticos naturales, temporales. & 37 & 25 \\
\hline 8 & Arrozales & Cuerpos de agua temporales asociados al cultivo de arroz. & 5 & 3 \\
\hline 9 & Embalses & Cuerpos de agua lénticos artificiales y de grandes dimensiones. & 10 & 5 \\
\hline 10 & $\begin{array}{l}\text { Balsas de riego y } \\
\text { estanques artificiales }\end{array}$ & $\begin{array}{l}\text { Cuerpos de agua lénticos artificiales de pequeñas dimensiones, que en } \\
\text { algunos casos pueden tener cierto proceso de naturalización. }\end{array}$ & 8 & 2 \\
\hline 11 & Cuevas & $\begin{array}{l}\text { Ecosistemas lóticos o lénticos en cavidades subterráneas, abiertas de } \\
\text { forma natural. }\end{array}$ & 5 & 5 \\
\hline
\end{tabular}




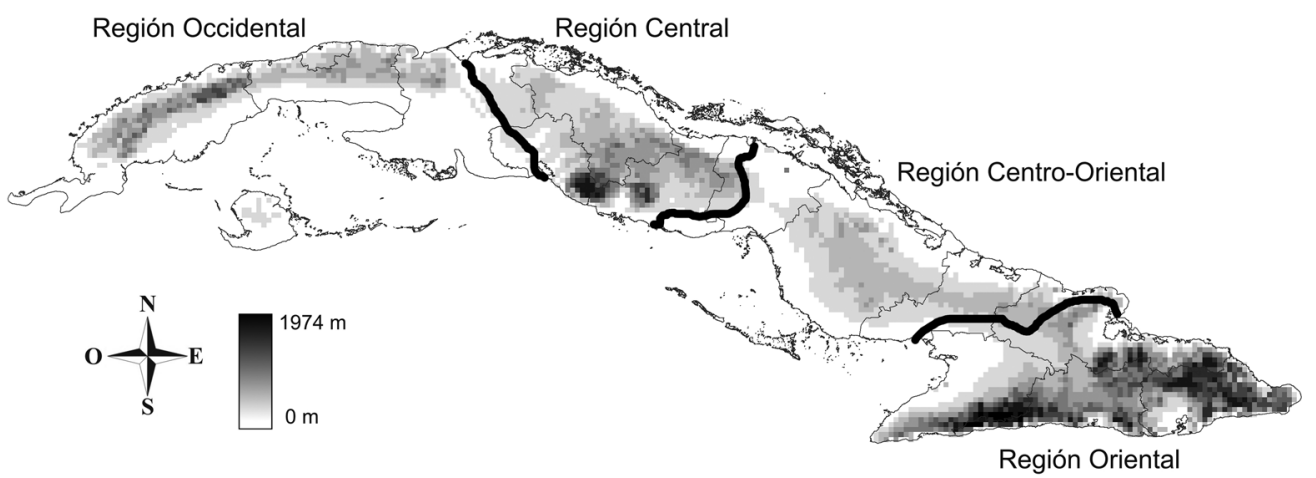

Fig. 1. Unidades espaciales (las cuatro principales regiones biogeográficas y las cuadrículas de 2.5 min) usadas para la evaluación de las vulnerabilidad de las especies de Dytiscidae. La escala de grises en las cuadrículas de 2.5 min representa el gradiente altitudinal.

Fig. 1. Spatial units (for the main four environmental regions and the 2.5 min squares) used for the assessment of the vulnerability of the species of Dytiscidae. Grey scale in the 2.5 squares represents the altitudinal gradient.

primera vez a escala nacional a partir de una adaptación de la metodología propuesta por Abellán, Sánchez-Fernández, Velasco y Millán (2005) y modificada por Sánchez-Fernández et al. (2008). Esta metodología se basa en un sistema de puntuación a partir de la combinación de seis criterios referidos a las características de las especies y de los hábitats que ocupan: distribución general, distribución en Cuba, rareza, persistencia, singularidad del hábitat y pérdida del hábitat.

Para cada uno de los criterios evaluados las especies pueden obtener cuatro valores (entre 0-3). Así, el valor final de vulnerabilidad de una especie es la suma de las puntuaciones de los seis criterios, y puede tomar valores entre cero y 18. Este valor total permite la ordenación de las especies en función de la prioridad de su conservación. A partir de esta metodología, los valores de vulnerabilidad se pueden agrupar en cuatro clases o categorías de vulnerabilidad: vulnerabilidad baja (0-4), media (5-8), alta (9-13) y muy alta (14-18) (Cuadro 2). El orden de prioridad para la conservación fue relacionado con las categorías de la Unión Internacional para la Conservación de la Naturaleza (UICN), a partir de las cuatro clases establecidas.

Criterios para la evaluación de la vulnerabilidad: Distribución General (DG). En este apartado las especies se clasificaron en cuatro tipos: Americana (A), aquellas especies presentes en la región Neártica y Neotropical; Neotropical $(\mathrm{N})$, aquellas presentes en el Caribe y Sudamérica; Antillana (AN), presentes en las Antillas Mayores y Menores; Endémicas de Cuba (E), aquellas presentes solo en Cuba (Cuadro 2). Esta clasificación biogeográfica se realizó según Morrone (2004).

Distribución en Cuba (DC). Las especies endémicas se clasifican según las siguientes categorías: Endemismo General: aparecen en las cuatros regiones biogeográficas (región Occidental, Central, Centro-Oriental y Oriental) de Cuba propuestas por Núñez (1989) (Fig. 1, Cuadro 3); Endemismo restringido: aparecen al menos en dos de las cuatro regiones; Endemismo estricto: con distribución limitada a una de las regiones, pero con presencia en al menos tres provincias dentro de esta región; Endemismo exclusivo: aquellas que aparecen solo dentro de una o dos provincias de la misma región (Cuadro 2).

Rareza de las especies (RE). La rareza de una especie según Rabinowitz, Cairnes y Dillon (1986) está determinada por la combinación de tres factores: especies con una distribución geográfica restringida (rareza geográfica), con baja abundancia (rareza demográfica) y que están asociadas a un estrecho espectro 
CUADRO 2

Criterios utilizados en el análisis de vulnerabilidad de las especies de Dytiscidae de Cuba y su rango de valores

TABLE 2

Criteria used in the vulnerability analysis of the Dytiscidae species from Cuba and their range of values

\begin{tabular}{|c|c|c|c|c|}
\hline \multirow{2}{*}{ Criterios } & \multicolumn{4}{|c|}{ Valores } \\
\hline & 0 & 1 & 2 & 3 \\
\hline $\begin{array}{l}\text { Distribución } \\
\text { General }\end{array}$ & Especies americanas & Especies neotropicales & Especies antillanas & Endemismos cubanos \\
\hline Distribución en Cuba & General & Restringido & Estricto & Exclusivo \\
\hline Rareza de las especies & $\begin{array}{l}\text { Ninguno de los siguientes criterios: } \\
\text { - Rango restringido (máximo tres } \\
\text { cuadrículas de } 2.5 \text { minutos) } \\
\text { - Baja abundancia (menos de tres } \\
\text { ejemplares en cualquier captura) } \\
\text { - Elevada especificidad de hábitat } \\
\text { (máximo dos tipos de hábitats) }\end{array}$ & $\begin{array}{l}\text { Uno de los criterios } \\
\text { antes listados }\end{array}$ & $\begin{array}{l}\text { Dos de los criterios } \\
\text { antes listados }\end{array}$ & $\begin{array}{l}\text { Tres de los criterios } \\
\text { antes listados }\end{array}$ \\
\hline Persistencia & $\begin{array}{l}\text { Última captura entre } 1990 \text { y } 2014 \text { ( } 24 \\
\text { años) }\end{array}$ & $\begin{array}{l}\text { Última captura entre } \\
1960 \text { y } 1990\end{array}$ & $\begin{array}{l}\text { Última captura entre } \\
1930 \text { y } 1960\end{array}$ & $\begin{array}{l}\text { Última captura entre } \\
1900 \text { y } 1930\end{array}$ \\
\hline $\begin{array}{l}\text { Singularidad del } \\
\text { hábitat }\end{array}$ & $\begin{array}{l}\text { Valores medios de rareza de los } \\
\text { tipos de hábitats entre } 0 \text { y } 0.75\end{array}$ & $\begin{array}{l}\text { Valores medios de } \\
\text { rareza de los tipos de } \\
\text { hábitats entre }>0.75 \\
\text { y } 1.50\end{array}$ & $\begin{array}{l}\text { Valores medios de } \\
\text { rareza de los tipos de } \\
\text { hábitats entre }>1.50 \\
\text { y } 2.25\end{array}$ & $\begin{array}{l}\text { Valores medios de } \\
\text { rareza de los tipos de } \\
\text { hábitats entre } \\
>2.25 \text { y } 3\end{array}$ \\
\hline $\begin{array}{l}\text { Pérdida del } \\
\text { hábitat }\end{array}$ & $\begin{array}{l}\text { Valores medios de vulnerabilidad } \\
\text { de los tipos de hábitats }<1\end{array}$ & $\begin{array}{l}\text { Valores medios de } \\
\text { vulnerabilidad } \\
\text { de los tipos de hábitats } \\
\text { entre } 1 \mathrm{y}<2\end{array}$ & $\begin{array}{l}\text { Valores medios de } \\
\text { vulnerabilidad } \\
\text { de los tipos de hábitats } \\
\text { entre } 2 \mathrm{y}<3\end{array}$ & $\begin{array}{l}\text { Valores medios de } \\
\text { vulnerabilidad } \\
\text { de los tipos de hábitats } \\
\text { entre } 3 \text { y } 4\end{array}$ \\
\hline
\end{tabular}

de hábitats (singularidad del hábitat) (Cuadro 2). En nuestro caso, fue considerado que una especie presenta distribución geográfica restringida en Cuba si aparece como máximo en tres cuadrículas de 2.5 × 2.5 min (Fig. 1). La abundancia se evaluó según el número de ejemplares encontrados para cada especie en cualquier muestreo. Aquellas especies con no más de 10 ejemplares capturados se consideran especies raras demográficamente. Por último, se determinó que una especie tenía especificidad de hábitat cuando apareció como máximo en dos de los 11 tipos de hábitats identificados (Cuadro 1).

Persistencia (P). Este apartado estuvo fundamentado en que las especies para las cuales no se dispone de citas recientes, pueden presentar mayor vulnerabilidad que aquellas que se han capturado regularmente. Para asignar a cada especie el valor de persistencia, se han distinguido cuatro intervalos de tiempo de aproximadamente 30 años a partir de 1900, excepto el más reciente entre 1990 y 2014 con 24 años.

Singularidad del hábitat (SH). Aquellas especies que ocupan hábitats muy singulares o raros geográficamente tendrán mayor probabilidad de desaparecer. Por ello, se evaluó este criterio solo para aquellas especies que suelen aparecer en un máximo de tres tipos de hábitats. En este caso, la singularidad de cada tipo de hábitat viene expresada en función del número de celdas de $2.5 \mathrm{~min}$ en las que aparece ese hábitat (Fig. 1).

La puntuación por singularidad de hábitats para cada especie tomó valores entre cero y tres: (0) hábitats muy comunes, que son aquellos que se han encontrado en más de 10 cuadrículas de $2.5 \mathrm{~min}$; (1) moderadamente comunes, presentes entre cinco y 10 cuadrículas; (2) 


\section{CUADRO 3}

Distribución de las especies de Dytiscidae en Cuba según las regiones biogeográficas propuestas por Núñez (1989)

TABLE 3

Distribution of the Dytiscidae species in Cuba according to the environmental regions biogeographic proposed by Núñez (1989)

\begin{tabular}{|c|c|c|c|c|c|}
\hline & \multirow[b]{2}{*}{ Especies } & \multicolumn{4}{|c|}{ Regiones biogeográficas } \\
\hline & & Occidental & Central & $\begin{array}{l}\text { Centro- } \\
\text { Oriental }\end{array}$ & Oriental \\
\hline \multirow[t]{9}{*}{ Laccophilinae } & *Laccodytes cobrinae Toledo, Megna \& Alarie, 2011 & & & & $\mathrm{x}$ \\
\hline & Laccomimus pumilio (LeConte, 1878) & $\mathrm{x}$ & & $\mathrm{x}$ & $\mathrm{x}$ \\
\hline & *Laccophilus alariei Megna, Deler-Hernández \& Challet, 2011 & $\mathrm{x}$ & & & \\
\hline & Laccophilus bifasciatus Chevrolat, 1863 & & & $\mathrm{x}$ & $\mathrm{x}$ \\
\hline & Laccophilus gentilis suavis Sharp, 1882 & & & $\mathrm{x}$ & $\mathrm{x}$ \\
\hline & Laccophilus ovatus zapotecus Zimmerman, 1970 & $\mathrm{x}$ & & & \\
\hline & Laccophilus proximus Say, 1823 & $\mathrm{x}$ & $\mathrm{x}$ & $\mathrm{x}$ & $\mathrm{x}$ \\
\hline & Laccophilus vacaensis vacaensis Young, 1953 & & & $\mathrm{x}$ & $\mathrm{x}$ \\
\hline & Laccophilus venustus Chevrolat, 1863 & $\mathrm{x}$ & $\mathrm{x}$ & & $\mathrm{x}$ \\
\hline \multirow[t]{9}{*}{ Copelatinae } & *Copelatus barbouri Young, 1942 & & & & $\mathrm{x}$ \\
\hline & Copelatus caelatipennis Chevrolat, 1863 & $\mathrm{x}$ & $\mathrm{x}$ & $\mathrm{x}$ & $\mathrm{x}$ \\
\hline & *Copelatus cordovai Megna \& Epler, 2012 & $\mathrm{x}$ & & $\mathrm{x}$ & $\mathrm{x}$ \\
\hline & Copelatus cubaensis Schaeffer, 1908 & $\mathrm{x}$ & $\mathrm{x}$ & $\mathrm{x}$ & $\mathrm{x}$ \\
\hline & *Copelatus danyi Megna \& Epler, 2012 & $\mathrm{x}$ & $\mathrm{x}$ & & \\
\hline & *Copelatus darlingtoni Young, 1942 & $\mathrm{x}$ & & & \\
\hline & Copelatus insolitus Chevrolat, 1863 & $\mathrm{x}$ & $\mathrm{x}$ & & $\mathrm{x}$ \\
\hline & *Copelatus montivagus Young, 1942 & & & & $\mathrm{x}$ \\
\hline & Copelatus posticatus (Fabricius, 1801) & $\mathrm{x}$ & $\mathrm{x}$ & & $\mathrm{x}$ \\
\hline \multirow[t]{19}{*}{ Hydroporinae } & Anodocheilus exiguus (Aubé, 1838) & $\mathrm{x}$ & & $\mathrm{x}$ & $\mathrm{x}$ \\
\hline & *Bidessonotus fallax Balfour-Browné, 1947 & $\mathrm{x}$ & & & \\
\hline & Bidessonotus browneanus Balfour-Browné, 1947 & $\mathrm{x}$ & & $\mathrm{x}$ & $\mathrm{x}$ \\
\hline & Bidessonotus caraibus (Chevrolat, 1863) & $\mathrm{x}$ & & & \\
\hline & Brachyvatus apicatus (Clark, 1862) & & $\mathrm{x}$ & & \\
\hline & Neoclypeodytes anasinus Miller, 2001 & $\mathrm{x}$ & & & $\mathrm{x}$ \\
\hline & Liodessus noviaffinis Miller, 1998 & & & & $\mathrm{x}$ \\
\hline & Neobidessus pullus floridanus (Fall, 1917) & $\mathrm{x}$ & & & \\
\hline & Hydrovatus caraibus Sharp, 1882 & & & $\mathrm{x}$ & $\mathrm{x}$ \\
\hline & Hydrovatus hornii Croth, 1873 & $\mathrm{x}$ & & & \\
\hline & Hydrovatus pustulatus (Melsheimer, 1844) & & & & $\mathrm{x}$ \\
\hline & *Desmopachria andreae Megna \& Sánchez-Fernández, 2014 & & $\mathrm{x}$ & & $\mathrm{x}$ \\
\hline & Desmopachria aspera Young, 1981 & $\mathrm{x}$ & $\mathrm{x}$ & & \\
\hline & Desmopachria darlingtoni Young, 1989 & & & & $\mathrm{x}$ \\
\hline & *Desmopachria glabella Young, 1981 & $\mathrm{x}$ & & & \\
\hline & *Desmopachria tarda Spangler, 1973 & & $\mathrm{x}$ & & $\mathrm{x}$ \\
\hline & Pachydrus obniger Chevrolat, 1863) & $\mathrm{x}$ & & $\mathrm{x}$ & $\mathrm{x}$ \\
\hline & Derovatellus floridanus Fall, 1932 & $\mathrm{x}$ & & & \\
\hline & Derovatellus lentus (Wehncke, 1876) & & & $\mathrm{x}$ & \\
\hline
\end{tabular}


CUADRO 3 (Continuación) / TABLE 3 (Continued)

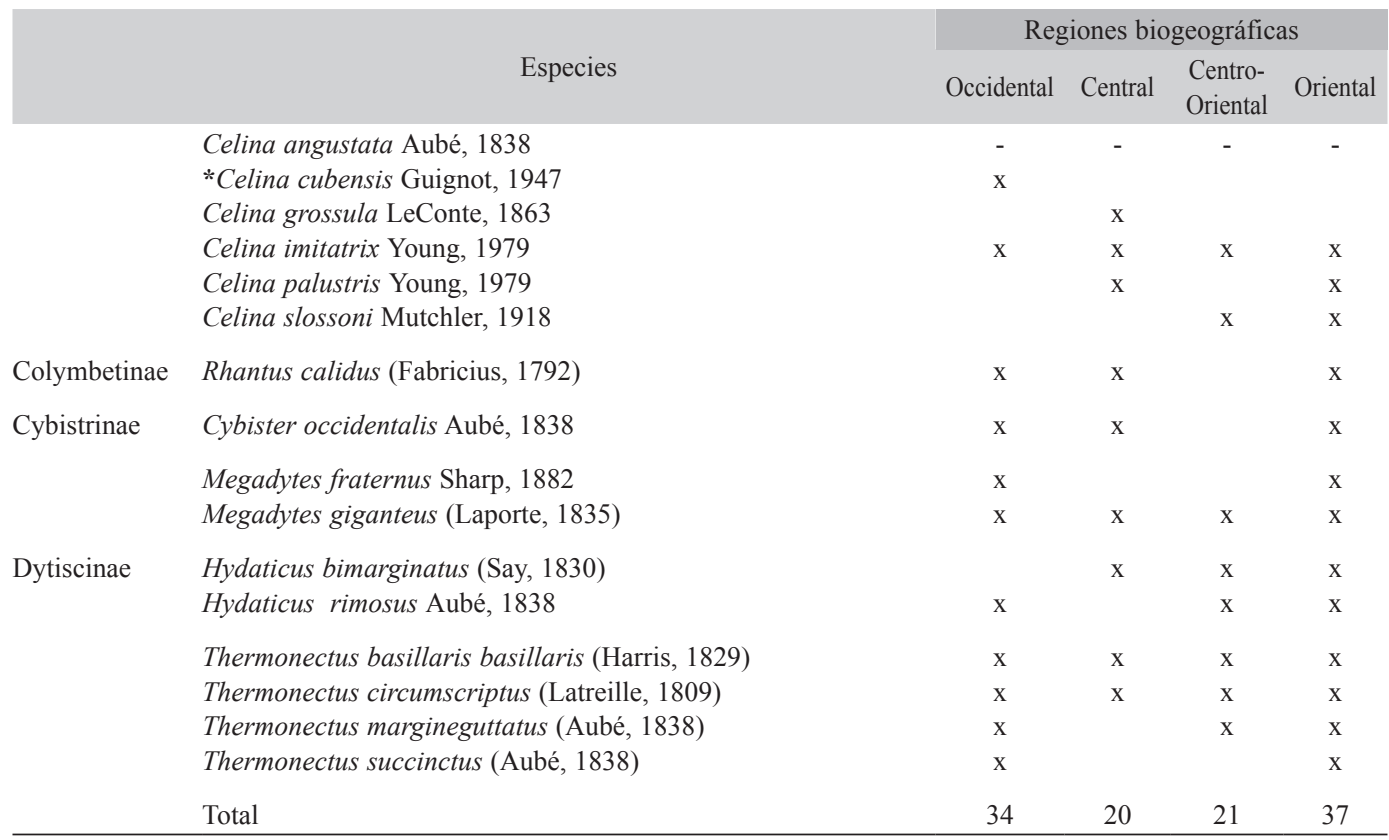

*especies endémicas/endemic species.

raros, presentes entre dos y cuatro cuadrículas; (3) muy raros, que se encuentran en una cuadrícula. La valoración de las especies fue realizada al sumar la puntuación de la rareza de cada uno de los tipos de hábitats en los que aparece una especie y dividiendo entre el número de tipos de hábitats ocupados. Así, por ejemplo, una especie que aparezca en dos tipos de hábitats, uno muy común (0) y otro muy raro (3), tendrá un valor de singularidad de hábitat de 1.5 calculado de la siguiente forma: $0+3$ $=3 ; 3 / 2=1.5$. Los valores para cada especie fueron integrados en cuatro categorías que se puntuaron de cero a tres (Cuadro 2).

Pérdida del hábitat $(\mathrm{PH})$. Este criterio hace referencia al riesgo de desaparición de los hábitats en los que aparece una especie, y fue determinado en función de los impactos que los afectan, siguiendo la misma metodología que Abellán et al. (2005) y considerando también lo propuesto en el IV Informe Nacional al Convenio Sobre la Diversidad Biológica en Cuba (Ministerio de Ciencia, Tecnología y Medio
Ambiente (Cuba), 2009). Para cada hábitat, estos impactos fueron evaluados y agrupados en cuatro clases:

1. Infraestructuras: son aquellos impactos producidos por alteraciones del cauce o llanura de inundación por la presencia de estructuras sólidas (obras de canalización, presas, puentes y cercanía a núcleos urbanos), o por extracciones de gravas.

2. Agrícola: en esta categoría se agrupan aquellos impactos derivados de la actividad agrícola en las cuencas de drenaje, tales como roturación de tierras para cultivos, derivación de agua para riego, contaminación difusa por fertilizantes y pesticidas.

3. Vertidos: vertidos al cauce del río, ya sean de redes de saneamiento o industrias.

4. Otros impactos: engloba los impactos ocasionados por el turismo, la cría intensiva de animales o la presencia de especies exóticas. 
CUADRO 4

Impactos que presentan los diferentes tipos de hábitats de las especies de Dytiscidae en Cuba

TABLE 4

Impacts of different types of habitats of Dytiscidae species in Cuba

\begin{tabular}{rlccccc}
$\mathrm{N}^{\mathrm{o}}$ & \multicolumn{1}{c}{ Tipos de hábitats } & Infraestructuras & Agrícola & Vertidos & Otros & Total \\
1 & Arroyos de cabecera & $\mathrm{x}$ & $\mathrm{x}$ & $\mathrm{x}$ & 1 \\
2 & Tramos medios de los ríos & $\mathrm{x}$ & $\mathrm{x}$ & $\mathrm{x}$ & $\mathrm{x}$ & 4 \\
3 & Tramos bajos de los ríos & $\mathrm{x}$ & $\mathrm{x}$ & $\mathrm{x}$ & $\mathrm{x}$ & 4 \\
4 & Estuarios de los ríos & & $\mathrm{x}$ & $\mathrm{x}$ & $\mathrm{x}$ & 3 \\
5 & Lagunas estuarinas & $\mathrm{x}$ & $\mathrm{x}$ & $\mathrm{x}$ & 3 \\
6 & Lagunas & & & $\mathrm{x}$ & 1 \\
7 & Charcas & & & & \\
8 & Cuevas & & & & \\
\hline
\end{tabular}

El siguiente paso fue calcular el valor de pérdida de hábitat para cada especie, en función de las amenazas que presentan los hábitats que ocupan (Cuadro 4). El valor viene dado por la suma de los diferentes impactos que presenta un hábitat determinado. No se evaluaron tres tipos de hábitats (arrozales, embalses, balsas de riego y estanques artificiales), ya que lejos de estar en peligro, actualmente están en expansión al ser hábitats artificiales.

Al igual que la singularidad del hábitat, solo se ha considerado la pérdida del hábitat para aquellas especies que aparecen en un máximo de tres tipos de hábitats. Para valorar las especies en función de la amenaza de los hábitats que ocupan, se sumó el valor de amenaza de cada tipo de hábitat en que aparece y se dividió este valor entre el número total de hábitats que ocupa. Por ejemplo, una especie que aparezca en dos tipos de hábitats, con un valor de amenaza de cero y dos respectivamente $(0+2=2 ; 2 / 2=1)$, obtendrá un valor de uno para este criterio (Cuadro 2). Los valores obtenidos para cada especie se integraron en cuatro categorías que se puntuaron desde 0 (aquellas especies que aparezcan en hábitats sin impactos), hasta tres (en aquellas especies que aparezcan solo en hábitats con el máximo número de impactos, ver Cuadro 2).

\section{RESULTADOS}

La familia Dytiscidae en Cuba está compuesta por seis subfamilias y 53 especies, de las cuales 12 son endémicas (Cuadro 3). La mayor riqueza registrada se encuentra en la región Oriental de Cuba con 37 especies, seguida por la Occidental (34), la Centro-Oriental (21) y la Central (20) (Cuadro 3). Las especies de distribución Antillana (18), en su mayoría (72\%), aparecieron en el Oriente de Cuba. Sin embargo, el género Celina tiene el mayor número de especies entre la región Central y Occidental (Cuadro 3, Fig. 1). De la misma manera, en el Occidente y el Oriente se encontraron siete especies endémicas, tres en el Centro y una en la Centro-Oriental (Cuadro 3).

De los 11 hábitats tipo diferenciados en la zona de estudio, los más comunes fueron las lagunas, que se encontraron en 57 localidades (o estaciones de muestreo), localizadas en 16 cuadrículas. Le siguen los arroyos de cabecera, con 42 localidades recogidas en 28 cuadrículas, lo cual muestra una mayor dispersión. Las charcas con 37 localidades y 25 cuadrículas, son el tercer hábitat tipo más frecuente y el segundo más disperso. Por otro lado, los hábitats tipo más raros fueron los estuarios de los ríos y lagunas estuarinas, apareciendo en sólo una localidad y una cuadrícula, respectivamente (Cuadro 1). Por otro lado, los hábitats más impactados fueron los tramos medios, bajos y estuarios de los ríos. Por el contrario, los arroyos de cabecera y las cuevas son los que se encuentran menos impactados.

El análisis de vulnerabilidad fue posible realizarlo a 50 de los 53 ditíscidos presentes en Cuba (Cuadro 5). Tres especies (Laccophilus 
CUADRO 5

Puntuación de vulnerabilidad de las especies de Dytiscidae de Cuba

TABLE 5Vulnerability score of the Dytiscidae species of Cuba

\begin{tabular}{|c|c|c|c|c|c|c|c|c|}
\hline \multirow{2}{*}{ Especies } & \multicolumn{8}{|c|}{ Criterios de evaluación de la vulnerabilidad } \\
\hline & DG & DC & RE & $\mathrm{P}$ & $\mathrm{SH}$ & $\mathrm{PH}$ & VV & Clase \\
\hline Laccodytes cobrinae & 3 & 3 & 3 & 0 & 0 & 1 & 10 & alta \\
\hline Laccophilus alariei & 3 & 3 & 2 & 0 & 0 & 2 & 10 & alta \\
\hline Copelatus barbouri & 3 & 3 & 3 & 3 & - & - & 12 & alta \\
\hline Copelatus darlingtoni & 3 & 3 & 3 & 3 & - & - & 12 & alta \\
\hline Desmopachria glabella & 3 & 3 & 3 & 1 & 0 & 1 & 11 & alta \\
\hline Neoclypeodytes anasinus & 2 & 0 & 2 & 0 & 0 & 3 & 7 & media \\
\hline Laccophilus bifasciatus & 2 & 0 & 0 & 0 & 0 & 3 & 5 & media \\
\hline Copelatus cordovai & 3 & 1 & 0 & 0 & 0 & 2 & 6 & media \\
\hline Copelatus danyi & 3 & 1 & 1 & 0 & 0 & 2 & 7 & media \\
\hline Copelatusmontivagus & 3 & 2 & 2 & 0 & 0 & 1 & 8 & media \\
\hline Bidessonotus caraibus & 1 & 0 & 2 & 1 & 0 & 3 & 4 & media \\
\hline Bidessonotus fallax & 3 & 1 & 1 & 0 & 0 & 2 & 7 & media \\
\hline Brachyvatus apicatus & 1 & 0 & 3 & 0 & 0 & 0 & 4 & media \\
\hline Liodessus noviaffinis & 0 & 0 & 3 & 1 & 0 & 3 & 7 & media \\
\hline Neobidessus pullus floridanus & 2 & 0 & 2 & 0 & 0 & 3 & 7 & media \\
\hline Hydrovatus pustulatus & 1 & 0 & 3 & 1 & 0 & 3 & 8 & media \\
\hline Desmopachria andreae & 3 & 1 & 1 & 0 & 0 & 2 & 7 & media \\
\hline Desmopachria aspera & 1 & 0 & 1 & 3 & - & - & 5 & media \\
\hline Desmopachria tarda & 3 & 1 & 0 & 0 & 0 & 2 & 6 & media \\
\hline Derovatellus lentus & 1 & 0 & 3 & 2 & 0 & 1 & 7 & media \\
\hline Celina grossula & 1 & 0 & 1 & 3 & - & - & 5 & media \\
\hline Celina palustris & 1 & 0 & 3 & 0 & 0 & 3 & 7 & media \\
\hline Cybister occidentalis & 1 & 0 & 2 & 1 & 0 & 2 & 6 & media \\
\hline Megadytes fraternus & 1 & 0 & 1 & 0 & 0 & 3 & 5 & media \\
\hline Megadytes giganteus & 1 & 0 & 2 & 2 & - & - & 5 & media \\
\hline Laccomimus pumilio & 1 & 0 & 2 & 0 & 0 & 1 & 4 & baja \\
\hline Laccophilus gentilis suavis & 1 & 0 & 0 & 0 & 0 & 0 & 0 & baja \\
\hline Laccophilus proximus & 0 & 0 & 0 & 0 & 0 & 0 & 0 & baja \\
\hline Laccophilus vacaensis vacaensis & 0 & 0 & 0 & 0 & 0 & 3 & 3 & baja \\
\hline Laccophilus venustus & 2 & 0 & 0 & 0 & 0 & 0 & 2 & baja \\
\hline Copelatus caelatipennis & 1 & 0 & 0 & 0 & 0 & 0 & 0 & baja \\
\hline Copelatus cubaensis & 1 & 0 & 0 & 0 & 0 & 3 & 4 & baja \\
\hline Copelatus insolitus & 1 & 0 & 0 & 0 & 0 & 0 & 1 & baja \\
\hline Copelatus posticatus & 1 & 0 & 0 & 0 & 0 & 0 & 1 & baja \\
\hline Anodocheilus exiguus & 1 & 0 & 0 & 0 & 0 & 0 & 1 & baja \\
\hline Bidessonotus browneanus & 2 & 0 & 0 & 0 & 0 & 0 & 2 & baja \\
\hline Hydrovatus caraibus & 1 & 0 & 0 & 0 & 0 & 0 & 1 & baja \\
\hline Hydrovatus hornii & 0 & 0 & 2 & 1 & 0 & 1 & 4 & baja \\
\hline Desmopachria darlingtoni & 1 & 0 & 1 & 0 & 0 & 1 & 3 & baja \\
\hline Pachydrus obniger & 3 & 1 & 0 & 0 & 0 & 0 & 4 & baja \\
\hline Derovatellus floridanus & 1 & 0 & 2 & 0 & 0 & 1 & 4 & baja \\
\hline Celina imitatrix & 0 & 0 & 0 & 0 & 0 & 0 & 0 & baja \\
\hline Celina slossoni & 0 & 0 & 0 & 0 & 0 & 0 & 0 & baja \\
\hline
\end{tabular}


CUADRO 5 (Continuación) / TABLE 5 (Continued)

\begin{tabular}{lcccccccc}
\multicolumn{1}{c}{ Especies } & \multicolumn{7}{c}{ Criterios de evaluación de la vulnerabilidad } \\
& DG & DC & RE & P & SH & PH & VV & Clase \\
Rhantus calidus & 0 & 0 & 0 & 0 & 0 & 0 & 0 & baja \\
Hydaticus bimarginatus & 0 & 0 & 0 & 0 & 0 & 2 & 2 & baja \\
Hydaticus rimosus & 1 & 0 & 0 & 0 & 0 & 2 & 3 & baja \\
Thermonectus basillaris basillaris & 0 & 0 & 0 & 0 & 0 & 0 & 0 & baja \\
Thermonectus circumscriptus & 1 & 0 & 0 & 0 & 0 & 0 & 1 & baja \\
Thermonectus margineguttatus & 1 & 0 & 0 & 0 & 0 & 2 & 3 & baja \\
Thermonectus succinctus & 1 & 0 & 0 & 0 & 0 & 0 & 1 & baja \\
Laccophilus ovatus zapotecus & - & - & - & - & - & - & - & - \\
Celina angustata & 1 & 0 & - & - & - & - & 1 & - \\
Celina cubensis & - & - & - & - & - & - & - & - \\
\hline
\end{tabular}

Distribución general (DG); Distribución en Cuba (DC); Rareza de la especie (RE); Persistencia (P); Singularidad del hábitat (SH); Pérdida de hábitat (PH); Valor de vulnerabilidad (VV); Sin datos (-).

General distribution (DG); Distribution in Cuba (DC).; Species rarity (RE); Persistence (P); Habitat singularity (SH); Loss of habitat (PH); Vulnerability value (VV); Without data (-).

ovatus zapotecus Zimmerman 1970, Celina angustata Aubé 1838 y Celina cubensis Guignot 1947) no han sido evaluadas ya que no existe información precisa sobre su distribución y preferencias de hábitat. Así, los resultados de este apartado señalan que 25 especies (48 \%) presentan vulnerabilidad baja, 20 (38 $\%)$ media, cinco especies (9\%) vulnerabilidad alta y ninguna presenta vulnerabilidad máxima (Cuadro 5). Las cinco especies más amenazadas son Laccodytes cobrinae, Laccophilus alariei, Copelatus barbouri, C. darlingtoni y Desmopachria glabella, todas endemismos cubanos (Cuadro 5).

\section{DISCUSIÓN}

Este estudio permitió evaluar que la distribución de la riqueza de ditíscidos, tiene mucha relación con el origen de la fauna cubana. Por ejemplo, el género de origen Neártico, Celina (Larson, Alarie, \& Roughley, 2000), está mejor representado en el occidente del país. Esto sugiere que la dirección de colonización también pudo ser desde el Norte. Resultados similares se han sugerido como vía de colonización desde las Antillas Mayores hasta la Florida para la familia Carabidae por Peck, Larivee y Browne (2005). Según estos autores el movimiento de las especies fue probablemente en el Pleistoceno cuando el nivel del mar era muy bajo. La similitud entre los ditíscidos cubanos con los de Sudamérica, justifica la hipótesis de que la mayoría de los taxa provienen de la conexión terrestre a través del arco de las Antillas. Existen varios géneros (Laccodytes, Laccomimus, Desmopachria y Thermonectus) de Dytiscidae cuyo centro de diversificación se ha establecido en la porción Central y Norte de Suramérica (Trémouilles, 1989; Miller, 2001; Toledo, Spangler, \& Balke, 2010; Toledo \& Michat, 2015) y luego se dispersaron alcanzando las Antillas (Peck, 2005, 2010, 2015). Esta dispersión se realizó por medio de la GAArlandia ("GreaterAntillesAvesRidgelandia"), conexión terrestre entre las Antillas Mayores y América del Sur (Iturralde-Vinent, 2006).

El análisis del estatus de conservación de ditíscidos permitió identificar aquellas especies que se encuentran más amenazadas. En total, se evaluó el $94 \%$ de las especies de la familia Dytiscidae citadas para Cuba. Nuestros resultados muestran que cinco de estas especies tienen un riesgo de extinción considerable, teniendo en cuenta que su distribución mundial está restringida a Cuba. No se encontró ninguna especie con grado de vulnerabilidad muy alto; sin embargo, en el caso de C. barbouri no se 
han incluido los valores por singularidad o pérdida de hábitat, ya que no se conocen con exactitud. Por tanto, en dependencia del tipo de hábitat en que aparezca en el futuro, pudiera aumentar su puntuación hasta ser incluida en la categoría de grado de vulnerabilidad muy alta. En este sentido, merece la pena focalizar los esfuerzos de conservación en estas seis especies. Así, se propone la inclusión de estos ditíscidos en futuros análisis de conservación, los cuales incrementarán los listados nacionales de especies amenazadas existentes (i.e., Amaro, 2012; González-Alonso, Rodríguez Schettino, Rodríguez, Mancina, \& Ramos García, 2012). Además, también se propone su inclusión en la Lista Roja de la UICN bajo la categoría de "Vulnerable", ya que al menos, cumplen con los criterios B1+2ab (i, ii, iii) de la UICN (IUCN, 2012), ya que se caracterizan por presentar poblaciones muy localizadas (tanto en extensión de la presencia como en área de ocurrencia), y con una disminución estimada tanto del rango de distribución como de la calidad del hábitat.

La metodología empleada contiene ciertos criterios y puntuaciones subjetivas como son los rangos de persistencia que varían según el nivel de conocimiento de cada región y el tamaño de las cuadrículas, que responden a un criterio de equilibrio entre precisión y realidad de los datos de colecciones y de la literatura. De igual forma, la UICN al emplear aspectos subjetivos en su metodología, dificulta aplicar algunos de los criterios y subcriterios a la mayoría de especies de insectos. Por ejemplo, el dinamismo de las poblaciones de insectos hace difícil conocer en qué medida varía realmente el tamaño poblacional de una especie, así como determinar con certeza los rangos de distribución (Cardoso et al., 2011). Todo lo anterior podría explicar el desequilibrio que existe entre el número de vertebrados e invertebrados incluidos en la lista roja de la UICN, ya que muchas especies están clasificadas como Datos Insuficientes, cuando en realidad presentan un notable riesgo de extinción (Cardoso, Erwin, Borges, \& New, 2011). De la misma manera, no se ha podido evaluar el grado de vulnerabilidad mediante un Análisis de Viabilidad Poblacional (Reed et al., 2002), basado en el análisis cuantitativo del riesgo de extinción para las poblaciones evaluadas; debido a que en la actualidad, no se dispone de la información detallada del tamaño de las poblaciones para realizar este estudio. El uso de una metodología adaptada al tipo de datos que se suele tener sobre insectos, como la empleada aquí, permite solucionar algunos de estos aspectos y avanzar en el conocimiento de su estatus de conservación (Abellán et al., 2005; Sánchez-Fernández et al., 2008).

La utilidad de la inclusión de extensos listados de especies de insectos poco vistosas en los catálogos nacionales o internacionales de especies amenazadas o incluso la Lista Roja de la UICN es bastante cuestionable, ya que se requiere de un importante conocimiento taxonómico para la identificación de estas especies, solamente al alcance de unos pocos especialistas. En este sentido, las medidas destinadas a la conservación de este tipo de especies, deben ir más dirigidas a la conservación del hábitat o las localidades en las que aparecen. Por lo tanto, la inclusión de estas especies en listas rojas de especies amenazadas no debe interpretarse como meros listados de especies que individualmente deberían estar protegidas atendiendo a su grado de vulnerabilidad, sino como listados de especies que pueden servir para localizar áreas interesantes de cara a su conservación y a proponer medidas de gestión dentro de ellas (Sánchez-Fernández et al., 2008). En este sentido, las áreas (Laguna Base Julio A. Mella, Punta del Este, Guanahacabibes, Sierra de Rosario, Norte de Imías, Monte Iberia y La Melba) en las que aparecen estas especies (ver Megna \& Sánchez-Fernández, 2016), no todas están dentro del Sistema Nacional de Áreas Protegidas de Cuba (CNAP, 2009). Por lo anterior, deben considerarse para su inclusión a la Laguna Base Julio A. Mella y el Norte de Imías, así como proponer medidas de gestión efectivas que eviten la alteración de los ecosistemas acuáticos.

La importancia del sistema de categorización utilizado en la presente investigación 
para clasificar los ditíscidos acuáticos de Cuba de acuerdo a su vulnerabilidad, junto con los resultados de los apartados anteriores, permite proponer una estrategia concreta a las instituciones encargadas de la gestión del medio natural. Ahora es posible señalar cuáles son las especies más amenazadas, donde están y cuáles son sus preferencias ambientales. Se recomienda realizar muestreos periódicos para actualizar el estado de las poblaciones, sobre todo en el caso de $C$. barbouri. Toda esta información es clave pues permite organizar las decisiones sobre la conservación biológica a partir de criterios objetivos y repetibles.

\section{AGRADECIMIENTOS}

Agradecemos a la fundación Alexander von Humboldt a través de la beca HERMES, a la Universidad de Castilla-La Mancha, el Fondo Social Europeo y al Departamento de Biología de la Universidad de Oriente.

\section{RESUMEN}

La diversidad de coleópteros acuáticos de Cuba se encuentra entre las más elevadas en el Caribe insular. Sin embargo, existe escasa información sobre los ditíscidos cubanos para identificar las especies y hábitats que requieren urgentes acciones de conservación. El objetivo de este estudio fue identificar los taxa amenazados de la familia Dytiscidae en Cuba según su grado de vulnerabilidad. Para ello se recopiló información sobre la distribución de todos los ditíscidos cubanos, incluyendo datos de la literatura, de colecciones y de muestreos realizados en el período comprendido entre los años 2000 y 2014. Además, fue evaluada la vulnerabilidad de las especies a partir de una metodología que combina seis criterios referentes a las características de las especies y los hábitats que ocupan. El análisis de vulnerabilidad fue posible realizarlo a $50 \mathrm{de}$ las 53 especies de ditíscidos (94\%) presentes en Cuba. Un total de cinco especies ( $9 \%$ ) presentan vulnerabilidad alta, $20(38 \%)$ media y 25 (48 \%) baja. Las cinco especies más amenazadas son Laccodytes cobrinae, Laccophilus alariei, Copelatus barbouri, C. darlingtoni, and Desmopachria glabella. Estas son endémicas de Cuba y se caracterizan por presentar poblaciones muy localizadas, poco abundantes, así como por una alta especificidad de hábitat. Por ello, se propone su inclusión en lista roja de la fauna de Cuba, así como en la Lista Roja de la UICN bajo la categoría de "Vulnerable". Además, no todas las áreas donde se encuentran estas especies pertenecen al Sistema Nacional de Áreas Protegidas de Cuba, por lo que recomendamos la inclusión de la Laguna Base Julio A. Mella y el Norte de Imías en este sistema. Así mismo, sugerimos la implementación de medidas de gestión efectivas que eviten o mitiguen la alteración de los ecosistemas acuáticos en los que aparecen las especies vulnerables.

Palabras clave: Adephaga; escarabajo buceador; especies amenazadas; UICN; biodiversidad.

\section{REFERENCIAS}

Abellán, P., Sánchez-Fernández, D., Velasco, J., \& Millán, A. (2005). Assessing conservation priorities for insects: status of water beetles in southeast Spain. Biological Conservation, 121, 79-90.

Amaro, V. S. (2012). Lista roja de la fauna cubana. La Habana, Cuba: AMA.

Benetti, C. J., \& Garrido, J. (2010). The influence of water quality and stream habitat on water betle asemblages in two rivers in northwest Spain. Vie Milieu, 60(1), 1-11.

Cardoso P., Borges, P. A.V., Triantis, K. A., Ferrández, M. A., \& Martín, J. L. (2011). Adapting the IUCN Red List criteria for invertebrates. Biological Conservation, 144, 2432-2440.

Cardoso, P., Erwin, T. L., Borges, P. A. V., \& New, T. R. (2011). The seven impediments in invertebrate conservation and how to overcome them. Biological Conservation, 144, 2647-2655.

Ceballos, G., Ehrlich, P. R., Barnosky, A. D., Garcia, A., Pringle, R. M., \& Palmer, T. M. (2015). Accelerated modern human-induced species losses: entering the sixth mass extinction. Science Advances, 1, e1400253.

Centro Nacional de Áreas Protegidas (CNAP). (2009). Sistema Nacional de Áreas Protegidas. Plan 2009-2013. La Habana, Cuba: Ministerio de Ciencia, Tecnología y Medio Ambiente.

Comisión Nacional de Nombres Geográficos. (2000). Diccionario Geográfico de Cuba. La Habana, Cuba, Oficina Nacional de Hidrografía y Geodesia: GEO. GEOCUBA.

González-Alonso, H., Rodríguez Schettino, L., Rodríguez, A., Mancina, C. A., \& Ramos García, I. (2012). Libro Rojo de los vertebrados de Cuba. La Habana, Cuba: Academia.

International Union for Conservation of Nature (IUCN). (2017). The IUCN Red List of Threatened Species. Version 2016.3. Retrieved from http://www. iucnredlist.org 
International Union for Conservation of Nature (IUCN). (2012). IUCN Red List Categories and Criteria: Version 3.1. $2^{\text {nd }}$ Ed. Gland, Switzerland and Cambridge, UK.

Iturralde-Vinent, M. (2006). Meso-Cenozoic Caribbean paleogeography: Implications for the historical biogeography of the region. International Geology Review, 48, 791-827.

Larson, D. J., Alarie Y., \& Roughley, R. E. (2000). Predaceous diving beetles (Coleoptera: Dytiscidae) of the Nearctic Region, with emphasis on the fauna of Canada and Alaska. Ottawa, Canadá: NRC Research Press.

Lundkvist, E., Ladin, J., Jackson, M., \& Svensson, C. (2003). Diving beetles (Dytiscidae) as predators of mosquito larvae (Culicidae) in field experiments and in laboratory tests of prey preference. Bulletin of Entomological Research, 93, 219-226.

Megna, Y. S., \& Epler, J. H. (2012). A review of Copelatus from Cuba, with the description of two new species (Coleoptera: Dytiscidae: Copelatinae). Acta Entomologica Musei Nationalis Pragae, 52, 383-410.

Megna, Y. S., \& Sánchez-Fernández, D. (2014). Desmopachria andreae a new species (Coleoptera: Dytiscidae: Hydroporinae) from Cuba, with the prediction of its geographic distribution. Zootaxa, 3753, 585-596.

Megna, Y. S., \& Sánchez-Fernández, D. (2016). Catálogo faunístico de la familia Dytiscidae (Coleoptera: Adephaga) en Cuba. Boletín de la Sociedad Entomológica Aragonesa (S.E.A.), 59, 159-180.

Megna, Y. S., Deler-Hernández, A., \& Challet, G. L. (2011). Description of a new species of Laccophilus Leach (Coleoptera: Dytiscidae), with notes on other Cuban species. The Coleopterists Bulletin, $65,213-226$

Miller, K. B. (2001). Descriptions of new species of Desmopachria Babington, 1841 (Coleoptera: Dytiscidae: Hydroporinae: Hyphydrini) with a reassessment of the subgenera and species groups and a synopsis of the species. The Coleopterists Bulletin, 55(2), 219-240.

Ministerio de Ciencia, Tecnología y Medio Ambiente (Cuba). (2009). IV Informe nacional al Convenio sobre la diversidad biológica. Cuba: Ministerio de Ciencia, Tecnología y Medio Ambiente.

Morrone, J. J. (2004). Panbiogeografía, componentes bióticos y zonas de transición. Revista Brasileira de Entomologia, 48, 149-162.

Núñez, A. (1989). Regiones naturales-antrópicas, p: XII.2.1. En: Instituto de Geografía de la Academia de Ciencias de Cuba e Instituto Cubano de Geodesia y Cartografía (Eds). Nuevo Atlas Nacional de Cuba. Madrid, España: Instituto Geográfico Nacional.
Peck, S. B. (2005). A checklist of the beetles of Cuba with data on distribution and bionomics (Insecta: Coleoptera). Arthropods of Florida and Neighboring Land Areas, 18, 1-241.

Peck, S. B. (2010). The beetles of the island of St. Vincent, Lesser Antilles (Insecta: Coleoptera); diversity and distributions. Insecta Mundi, 144, 1-77.

Peck, S. B. (2015). The beetles of the Lesser Antilles (Insecta, Coleoptera): diversity and distributions. Insecta Mundi, 0460, 1-360.

Peck, S. B., Larivee M., \& Browne J. (2005). Biogeography of ground beetles of Florida (Coleoptera: Carabidae): The peninsula effect and beyond. Conservation Biology and Biodiversity. Annals of the Entomological Society of America, 98(6), 951-959.

Rabinowitz, D., Cairnes, S., \& Dillon, T. (1986). Seven forms of rarity and their frequency in the flora of the British Isles. In M. E. Soulé (Ed.), Conservation Biology: The Science of Scarcity and Diversity (pp.182-204). Sunderland, Massachusetts, EUA: Sinauer Associates.

Reed, J. M., Mills, L. S., Dunning, J. B., Menges, E. S., McKelvey, K. S., Frye, R., Beissinger, S. R., Anstett, M., \& Miller, P. (2002). Emerging issues in Population Viability Analysis. Biological Conservation, 16, 7-19.

Sánchez-Fernández, D., Bilton, D. T., Abellán, P., Ribera, I., Velasco J., \& Millán, A. (2008). Are the endemic water beetles of the Iberian Peninsula and the Balearic Islands effectively protected? Biological Conservation, 141, 1612-1627.

Strayer, D. L. (2006). Challenges for freshwater invertebrate conservation. Journal of the North American Benthological Society, 25, 271-287.

Toledo, M., Megna, Y. S., \& Alarie, Y. (2011). Description of a new species of Laccodytes Régimbart, 1895 (Coleoptera, Dytiscidae, Laccophilinae) from Cuba. Zootaxa, 2792, 63-67.

Toledo, M., \& Michat, M. C. (2015). Description of Laccomimus gen. n. and eleven new species from the Neotropical region (Coleoptera, Dytiscidae, Laccophilinae). Zootaxa, 3990(3), 301-354

Toledo, M., Spangler, P. J., \& Balke, M. (2010). Taxonomic revision of the Neotropical diving beetles genus $\mathrm{Lac}$ codytes Régimbart, 1895 (Coleoptera: Dytiscidae). Zootaxa, 2347, 37-58.

Trémouilles, E. (1989). Contribución para conocimiento del género Thermonectus Dejean en la Argentina y áreas limítrofes (Coleoptera: Dytiscidae). Revista de la Sociedad Entomológica Argentina, 46(1-4), 95-115. 